\title{
Estradiol Vaginal Ring
}

National Cancer Institute

\section{Source}

National Cancer Institute. Estradio/ Vaginal Ring. NCI Thesaurus. Code C78471.

A flexible elastomer ring containing 17 beta-estradiol used for estrogen replacement. Upon vaginal insertion, estradiol vaginal ring releases a consistent low-dose of estrogen which binds to and activates nuclear receptors in estrogen-responsive tissues. By increasing the amount of estradiol locally, symptoms of vaginal dryness or decreased sexual interest may improve. 17 beta-estradiol is the major naturally occurring estrogen produced in the ovaries of premenopausal women. 